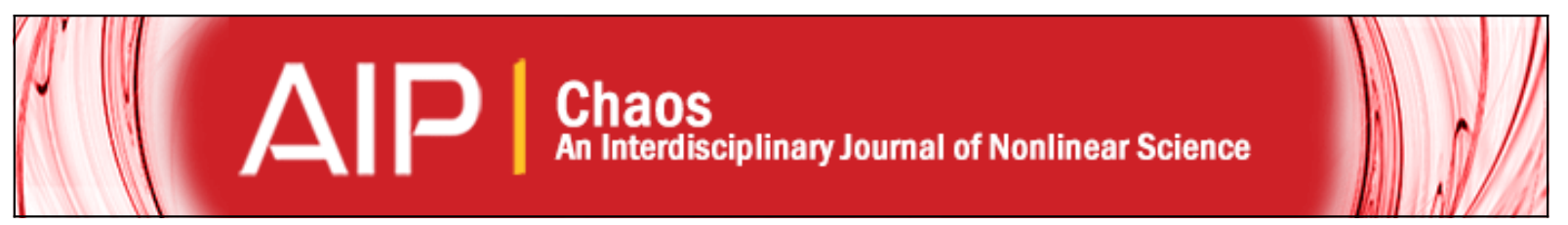

\title{
The temporal patterns of disease severity and prevalence in schistosomiasis
}

Manuela Ciddio, Lorenzo Mari, Marino Gatto, Andrea Rinaldo, and Renato Casagrandi

Citation: Chaos: An Interdisciplinary Journal of Nonlinear Science 25, 036405 (2015); doi: 10.1063/1.4908202

View online: http://dx.doi.org/10.1063/1.4908202

View Table of Contents: http://scitation.aip.org/content/aip/journal/chaos/25/3?ver=pdfcov

Published by the AIP Publishing

\section{Articles you may be interested in}

A support vector machine classifier reduces interscanner variation in the HRCT classification of regional disease pattern in diffuse lung disease: Comparison to a Bayesian classifier

Med. Phys. 40, 051912 (2013); 10.1118/1.4802214

Spatio-temporal Modeling of Mosquito Distribution

AIP Conf. Proc. 1404, 162 (2011); 10.1063/1.3659916

Automated segmentation of lungs with severe interstitial lung disease in CT

Med. Phys. 36, 4592 (2009); 10.1118/1.3222872

Automatic analysis of medial temporal lobe atrophy from structural MRIs for the early assessment of Alzheimer disease

Med. Phys. 36, 3737 (2009); 10.1118/1.3171686

Bayesian Estimation of Fish Disease Prevalence from Pooled Samples Incorporating Sensitivity and Specificity AIP Conf. Proc. 659, 39 (2003); 10.1063/1.1570533

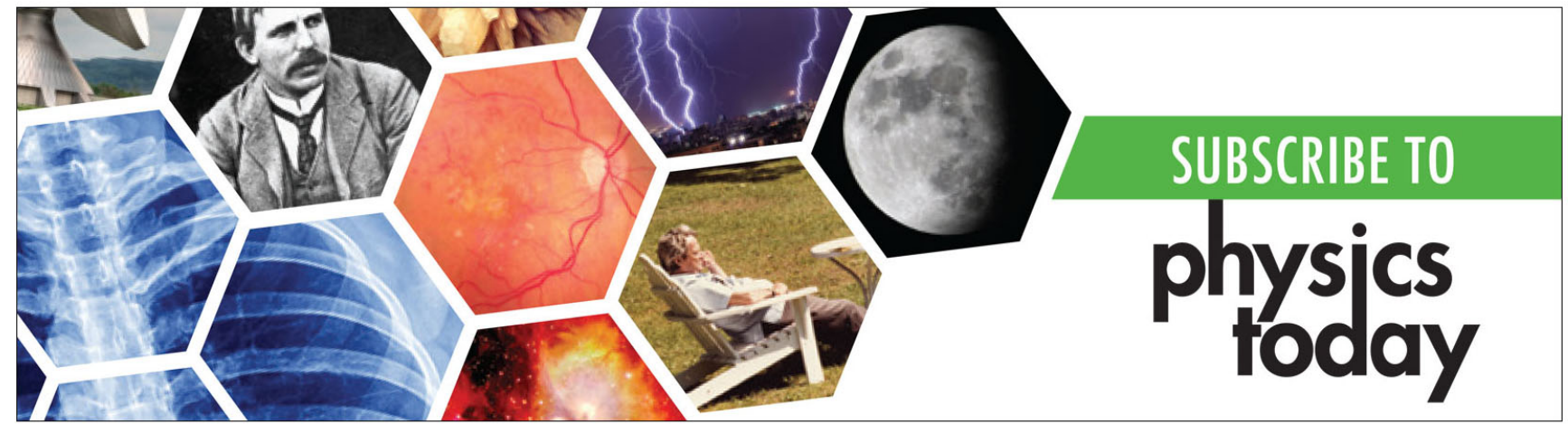




\title{
The temporal patterns of disease severity and prevalence in schistosomiasis
}

\author{
Manuela Ciddio, ${ }^{1}$ Lorenzo Mari, ${ }^{1,2}$ Marino Gatto, ${ }^{1, a)}$ Andrea Rinaldo, ${ }^{2,3}$ \\ and Renato Casagrandi $\left.{ }^{1, b}\right)$ \\ ${ }^{1}$ Dipartimento di Elettronica, Informazione e Bioingegneria, Politecnico di Milano, Via Ponzio 34/5, 20133 \\ Milano, Italy \\ ${ }^{2}$ Laboratory of Ecohydrology, ECHO/IIE/ENAC, École Polytechnique Fédérale de Lausanne, Station 2, \\ 1015 Lausanne, Switzerland \\ ${ }^{3}$ Dipartimento di Ingegneria Civile, Edile ed Ambientale, Università di Padova, Via Loredan 20, \\ 35131 Padova, Italy
}

(Received 30 October 2014; accepted 2 February 2015; published online 17 February 2015)

\begin{abstract}
Schistosomiasis is one of the most widespread public health problems in the world. In this work, we introduce an eco-epidemiological model for its transmission and dynamics with the purpose of explaining both intra- and inter-annual fluctuations of disease severity and prevalence. The model takes the form of a system of nonlinear differential equations that incorporate biological complexity associated with schistosome's life cycle, including a prepatent period in snails (i.e., the time between initial infection and onset of infectiousness). Nonlinear analysis is used to explore the parametric conditions that produce different temporal patterns (stationary, endemic, periodic, and chaotic). For the time-invariant model, we identify a transcritical and a Hopf bifurcation in the space of the human and snail infection parameters. The first corresponds to the occurrence of an endemic equilibrium, while the latter marks the transition to interannual periodic oscillations. We then investigate a more realistic time-varying model in which fertility of the intermediate host population is assumed to seasonally vary. We show that seasonality can give rise to a cascade of period-doubling bifurcations leading to chaos for larger, though realistic, values of the amplitude of the seasonal variation of fertility. (C) 2015 AIP Publishing LLC.
\end{abstract}

[http://dx.doi.org/10.1063/1.4908202]

In recent years, mathematical modeling has become an extremely useful tool to understand and analyze the temporal evolution of infections, also helpful in supporting the development of control strategies. In this work, we propose stability and bifurcation analyses of a nonlinear model of schistosomiasis, a waterborne parasitic disease caused by a snail-transmitted trematode. Our aim is to explain the interannual oscillations that characterize schistosomiasis prevalence in endemic regions, introducing some innovation in modeling the intermediate snail hosts. The analysis is based on a system of differential equations that describe the dynamics of schistosomes (adult parasites and larval stages), snails (intermediate host), and humans (definitive host). The main contributions of the present study are (a) to find the equilibria of the system under different parametric conditions and to study their stability, (b) to provide a nearly complete bifurcation analysis for the model, and (c) to investigate a more realistic version of the model accounting for time-varying ecological dynamics of the snail host.

\section{INTRODUCTION}

Schistosomiasis, also known as bilharziasis, is one of the commonest waterborne diseases. It is a major parasitic

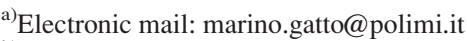

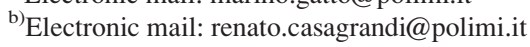

infection in many areas of the developing world, affecting more than $200 \times 10^{6}$ individuals in 74 countries and putting at risk about $600 \times 10^{6}$ people worldwide. ${ }^{1}$ Schistosomiasis is considered one of the Neglected Tropical Diseases (NTDs) and in terms of impact is second only to malaria as the most devastating parasitic disease. ${ }^{2}$ Its burden is disproportionately concentrated in Africa. According to the World Health Organization, many control programs are available which can successfully eliminate the disease. However, schistosomiasis remains a major cause of mortality and morbidity in a number of countries, notably those of sub-Saharan Africa, ${ }^{2}$ but also in some areas of Asia and Latin America. ${ }^{1}$ In particular, in sub-Saharan Africa schistosomiasis contributes to more than 200000 deaths annually, with an estimated disability-adjusted life years (DALYs, i.e., the number of years lost due to ill-health, disability, or early death) of $4.5 \times 10^{6} .2$

It is estimated that $120 \times 10^{6}$ of infected individuals worldwide are symptomatic, with $20 \times 10^{6}$ developing severe symptoms from the disease. Although the disease has a low mortality rate, the related morbidity caused by this disease can inflict a heavy health burden on high-prevalence communities. The largest intensity of infection is usually observed in children, who are especially vulnerable and, if not treated, suffer chronic consequences into adulthood. Typically, parasites inside human tissues induce a response that causes local and systemic pathological effects ranging from anaemia, impaired growth and cognitive development, and decreased physical fitness, to organ-specific effects such 
as fibrosis of the liver, bladder cancer, and urogenital inflammation. ${ }^{3,4}$

Schistosomiasis is caused by trematode parasites belonging to the genus Schistosoma. Most human infections are caused by three major species: Schistosoma mansoni, S. haematobium, or $S$. japonicum. ${ }^{2}$ These parasites need as intermediate hosts certain types of freshwater snails belonging to the genus Biomphalaria for S. mansoni, Bulinus for $S$. haematobium, and Oncomelania for S. japonicum. The geographical distribution of schistosomes is in fact defined by the specific range of snail host habitat. Thus S. haematobium and S. mansoni occur in both Africa and the Middle East, whereas only S. mansoni is present in the Americas. S. japonicum is localized in Asia, primarily the Philippines and China. ${ }^{3}$

The infectious form of the parasite (for humans) is a freely swimming larval stage known as cercaria. Cercariae emerge from the snail, hence contaminating water, and can infect humans penetrating their skin when they come into contact with contaminated freshwater. ${ }^{2}$ Inside the human body, cercariae shed their tails and become schistosomula, the maturing larvae of the parasite. These larvae migrate through the body and need about 5-7 weeks before becoming sexually mature adults. ${ }^{3}$ The adult male and female worms colonize human blood vessels, where they can live for years, mating and producing hundreds to thousands of fertilized eggs daily. The eggs can either leave the body of the host by being shed in the environment through faeces (S. mansoni, S. japonicum) or urine (S. haematobium), or become trapped inside the human host tissues. The severity and complexity of the pathology of schistosomiasis are related to the quantity of these encysted eggs. ${ }^{2}$ The eggs released out of the human body that reach freshwater can hatch into larvae called miracidia, the parasite larval form that is infectious for snail hosts. In the snail, miracidia undergo asexual replication for $4-6$ weeks, ${ }^{3}$ then the snail becomes infective and starts releasing tens of thousands of cercariae into the water. The time between initial snail infection and onset of infectiousness is the so-called prepatent period. ${ }^{3}$ Cercariae can survive in freshwater for 1-3 days, ${ }^{3}$ then they need to find a human host to complete the parasite's life cycle (Fig. 1).
The occurrence of schistosomiasis displays both interand intra-annual variability (see, e.g., Fig. 2 for some sample patterns from Limpopo Province, South Africa, ${ }^{5}$ and Wonji, Ethiopia $^{6}$ ). While intra-annual oscillations can be explained by the strong seasonal fluctuations of snail demography, interannual fluctuations, which can be more or less wide, may be caused by nonlinearities in demographic and epidemiological mechanisms.

Mathematical modeling of disease dynamics has proved to be a useful tool in many human infections, in particular, to understand the transmission characteristics of parasitic diseases in order to develop and evaluate the effects of control programs, $^{7-9}$ and to make predictions on the effects of different intervention options, ${ }^{10-12}$ also exploring control strategies targeted at high-risk behavioral groups. ${ }^{13,14}$ Transmission models of schistosomiasis have been in existence since the 1960s. ${ }^{15,16}$ These models are generally based on some limiting assumptions (e.g., homogeneous population, ${ }^{16}$ single host population and one parasite stage ${ }^{17}$ ). More recent studies have tried to relax these assumptions, e.g., by accounting for the demographic dynamics of human and snail populations, or by including some additional aspects (e.g., infection age of snails, ${ }^{18,19}$ parasite's mating structure and multiple resistant schistosome strains ${ }^{20}$ ). They usually neglect the dynamics of the intermediate life stages of the parasite, thus reducing model complexity.

In this paper, we want to explore the mechanisms that drive the temporal variability of schistosomiasis severity and prevalence using a model of intermediate complexity, which allows a thorough bifurcation analysis. Our innovation concerns specifically snail dynamics. In fact, the presence of a prepatent period in snails, $3,18,19$ in which the infection is ongoing but release of cercariae cannot be detected, suggests the division of snails into three classes, according to their infection stage, similarly to a classical Susceptible-ExposedInfectious epidemiological model. This model is less complex than those including the snails' infection age, ${ }^{18,19}$ yet it is fairly realistic and simple enough to allow an analytical investigation of the link between the ecology of the snails and the variability of typical disease patterns observed in many endemic regions.

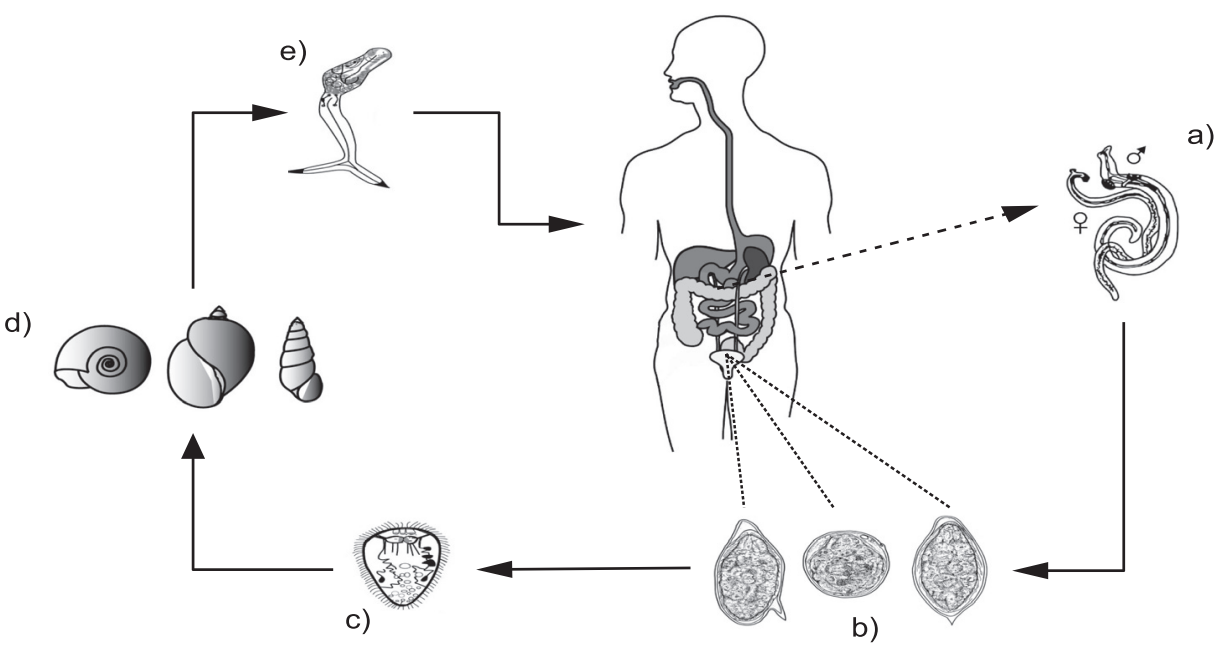

FIG. 1. Schistosoma life cycle. (a) Paired adult worms (larger male enfolding slender female). (b) Eggs (left to right, S. mansoni, S. japonicum, S. haematobium). (c) Ciliated miracidium. (d) Intermediate host snails (left to right, Biomphalaria, Bulinus, Oncomelania). (e) Cercaria. 

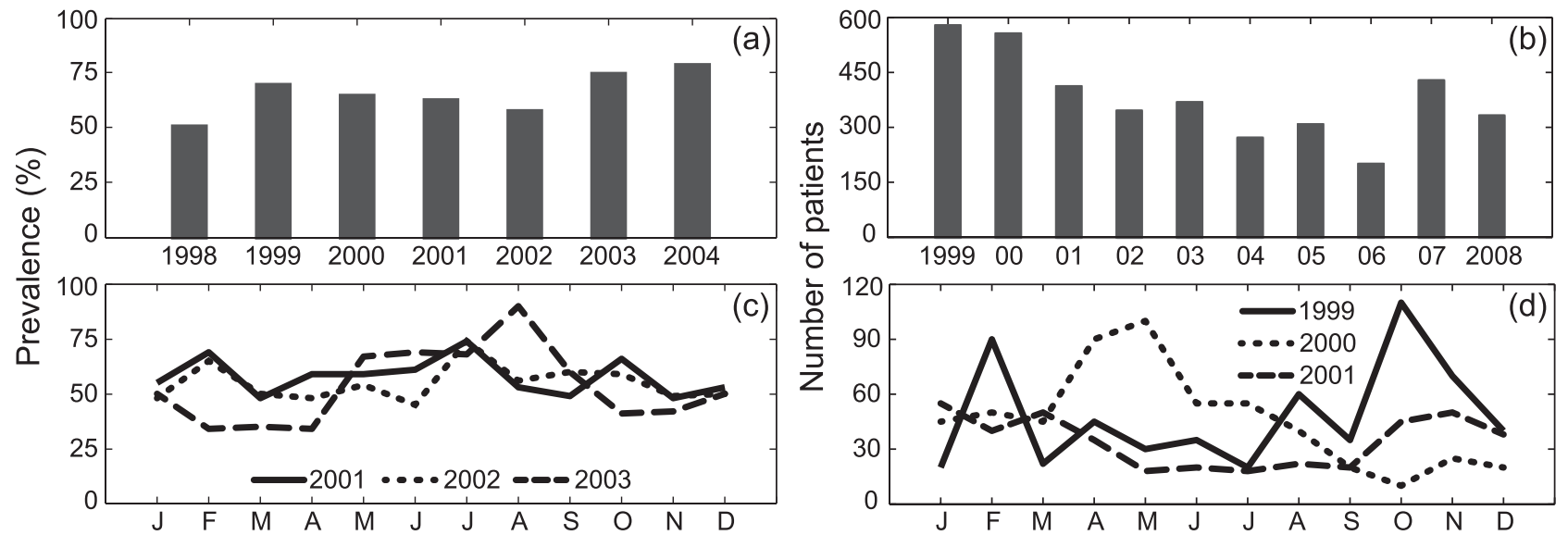

FIG. 2. Yearly and monthly occurrence of schistosomiasis in South Africa and Ethiopia. (a) Overall yearly prevalence of urinary schistosomiasis amongst patients attending the main hospitals in the Vhembe district of Limpopo Province, South Africa, between 1998 and 2004. (b) Overall yearly number of S. mansoni patients between 1999 and 2008 from the only hospital in Wonji, Ethiopia. (c) Occurrence of S. haematobium in urine samples submitted for urinary tract infections to the laboratory of the Vhembe district hospitals between 2001 and 2003. (d) Monthly data of the number of S. mansoni patients in Wonji between 1999 and 2001. Data elaborated from Samie et al. ${ }^{5}$ (panels (a) and (c)) and Xue et al..$^{6}$ (panels (b) and (d)).

\section{THE MODEL}

\section{A. Model formulation}

Our work is based on a system of differential equations that describe the dynamics of humans, schistosomes, and snails, according to the ecological and epidemiological mechanisms that characterize human schistosomiasis transmission (Fig. 1). The model can be considered as the combination of two sub-systems describing, respectively, the dynamics of humans and adult worms in their tissues, and snails dynamics.

The first sub-system is governed by two differential equations that describe the dynamics of human hosts $N$ and of the adult form of parasites $P$. The man-schistosome interaction is modeled as a macroparasitic infection ${ }^{7}$

$$
\left\{\begin{array}{l}
\dot{N}=\mu_{H}(H-N)-\alpha P \\
\dot{P}=\beta C N-\left(\mu_{H}+\mu_{P}+\alpha\right) P-\alpha \frac{k+1}{k} \frac{P^{2}}{N} .
\end{array}\right.
$$

Human hosts are assumed to be characterized by a constant recruitment $\mu_{H} H$ (with $H$ being the community size), and a natural death rate $\mu_{H}$. We assume that parasites induce mortality proportionally to their number within each human host, i.e., proportionally to the mean worm burden $P / N$. With $\alpha$ being a constant determining the pathogenicity of the parasite to the human host, ${ }^{17}$ the total losses due to diseaseinduced mortality of humans thus are

$$
\alpha \frac{P}{N} N=\alpha P .
$$

Parasites' recruitment is proportional to the number of cercariae $C$ in the environment and to an infection rate $\beta$ that includes several aspects of survival and maturation of cercariae inside and outside the human hosts. Since the timescale of the free-living larval stages is much shorter than that of the mature worm in the human host and the developing stage in snails, cercariae are assumed to be proportional to the total number $I$ of infectious shedding snails, ${ }^{7,18} C=r I$, where $r$ represents the number of cercariae released by one infected snail multiplied by the residence time of cercariae in water. The death rate of parasites within the human host population has three components. First, there are losses due to natural host mortality $\left(\mu_{H} P\right)$. Second, there is a component linked to parasite mortality within the host. Assuming an intrinsic death rate $\mu_{P}$, these losses make a contribution of $\mu_{P} P$. Third, there are losses due to disease-induced mortality of humans. Since macroparasites are almost always unevenly distributed across their host populations, negative binomial is often used for modeling parasite differences in load among different individuals. ${ }^{18}$ Thus, these losses can be written as a function of the clumping parameter $k$, which gives an inverse measure of the degree of aggregation of the parasites within the hosts ${ }^{17}$

$$
\alpha \frac{P}{N} N\left(1+\frac{k+1}{k} \frac{P}{N}\right)=\alpha P+\alpha \frac{k+1}{k} \frac{P^{2}}{N} .
$$

The second sub-system describes the dynamics of snails. In order to consider the prepatent period after initial infection, snail dynamics is described via a compartmental SEIlike model that introduces a delay between infection and onset of infectiousness. The snail population is thus divided into Susceptible, Exposed, and Infectious individuals, according to their ability to be infected and to infect (i.e., their ability to release cercariae after initial infection). The system has the following form:

$$
\left\{\begin{array}{l}
\dot{S}=b(S, E, I)-\mu_{S} S-\rho M S \\
\dot{E}=\rho M S-\left(\mu_{S}+d_{S}\right) E-\delta E \\
\dot{I}=\delta E-\left(\mu_{S}+d_{S}\right) I
\end{array}\right.
$$

in which $b$ is the recruitment rate of susceptible snails. Other studies have shown that snail populations are regulated by density-dependent mechanisms ${ }^{21}$ and that the introduction of a nonlinear recruitment function seems to play an important role in generating realistic patterns of schistosome infections. ${ }^{18}$ Here, we assume that snails are born uninfected 
according to a logistic recruitment function and that infected snails (both exposed and infectious) are unable to reproduce, thus

$$
b(S, E, I)=\nu S[1-\gamma(S+E+I)],
$$

where $\nu$ is the intrinsic natality rate and $\gamma$ captures the effect of density dependence among snails. In addition to a natural death rate $\mu_{S}$, infected snails are also subject to a diseaseinduced death rate $d_{S}$. We assume that a population of uninfected snails is always viable, namely, $\nu>\mu_{S}$. Recruitment of exposed snails depends on the infection rate $\rho$ and the number of miracidia $M$ in the aquatic environment. Miracidia, being short-lived, are assumed to be proportional to the number of adult parasites through the per capita egg laying rate $l_{P}$ of adult parasites and the residence time of miracidia in water $\tau_{M}$, so that $M=l_{P} \tau_{M} P$. For simplicity of notation, we introduce $\chi=\rho l_{P} \tau_{M}$ to aggregate the various intermediate steps involved in snail infection. After infection, snails enter the Exposed compartment (they are infected but not yet infectious, i.e., they do not release cercariae). After a prepatent period of average duration $1 / \delta$, they complete the cycle entering into the Infectious class and starting to release cercariae.

Combining (1)-(5), the full model that describes schistosomiasis dynamics is represented by the following system of nonlinear differential equations:

$$
\left\{\begin{array}{l}
\dot{N}=\mu_{H}(H-N)-\alpha P \\
\dot{P}=\beta r I N-\left(\mu_{H}+\mu_{P}+\alpha\right) P-\alpha \frac{k+1}{k} \frac{P^{2}}{N} \\
\dot{S}=\nu S[1-\gamma(S+E+I)]-\mu_{S} S-\chi P S \\
\dot{E}=\chi P S-\left(\mu_{S}+d_{S}\right) E-\delta E \\
\dot{I}=\delta E-\left(\mu_{S}+d_{S}\right) I .
\end{array}\right.
$$
Table I.

\section{B. Parameter estimation}

Parameters associated with infection of human hosts are usually quite difficult to estimate. Here, part of the parameters is derived from literature, and part is allowed to vary because their values are uncertain or dependent upon exogenous conditions. In these latter cases, sensitivity and bifurcation analyses are usually the best approach to identify parameters able to produce realistic results ${ }^{22}$ and to evaluate the effects of combined changes in the values of specific parameters. ${ }^{18}$ This approach has been used also in previous studies on other schistosomiasis models. ${ }^{23,24}$

We use a human population of size $H=1000$ individuals with a life expectancy of $70 \mathrm{yr}\left(\mu_{H}=4 \times 10^{-5} /\right.$ day $)$. The life expectancy of adult parasites is $5 \mathrm{yr}^{19,25}\left(\mu_{P}=5.5 \times\right.$ $10^{-4} /$ day). Parasite-induced mortality in the human host is considered to be negligible (for example, the value estimated for an endemic area in Sudan was $\alpha=1.1 \times 10^{-7} /$ day. $^{26}$ Therefore, we first set $\alpha=0$ to make the model amenable to analytical investigation. This hypothesis is later relaxed, by allowing $\alpha$ to assume values $>0$. In this latter case, the clumping parameter of the negative binomial distribution of parasites within human hosts is set to the value estimated in a previous study of a similar model, ${ }^{19} k=0.243$.

As for snails, we consider an average lifetime of $1 \mathrm{yr}^{19}$ for uninfected snails $\left(\mu_{S}=2 . \times 10^{-3} /\right.$ day $)$ and 2 months ${ }^{19}$ for infected snails $\left(d_{S}=1.7 \times 10^{-2} / \mathrm{day}\right)$. The average duration of the prepatent period is assumed to be about 2 weeks ${ }^{19,25}\left(\delta=6.7 \times 10^{-2} /\right.$ day $)$, and the average number of cercariae released by one infected snail to be $350 /$ day, ${ }^{19}$ thus $r=700$ cercariae/snail (with average residence time of 2 days $\left.^{3}\right)$. We assume a carrying capacity of $\approx 100$ snails $/ \mathrm{m}^{2}$ in absence of the parasite ${ }^{27}\left(\gamma=10^{-2} \mathrm{~m}^{2} /\right.$ snail $)$. The intrinsic natality rate of snails is strongly dependent on the environmental conditions ${ }^{28}$ and exposition to schistosomes. ${ }^{21} \mathrm{We}$ assume that each snail produces at most 180 eggs in 10 weeks ${ }^{21}$ and, with a hatching rate of about $30 \%$, we set $\nu=0.7 /$ day. All parameters are listed in Table I.

\begin{tabular}{|c|c|c|c|c|}
\hline Name & Description & Value & Units & References \\
\hline$N$ & Number of human hosts & & Individual & \\
\hline$P$ & Number of adult parasites & & Parasite & \\
\hline$S$ & Density of susceptible snails & & Snail $\times \mathrm{m}^{-2}$ & \\
\hline E & Density of exposed snails & & Snail $\times \mathrm{m}^{-2}$ & \\
\hline$I$ & Density of infectious snails & & Snail $\times \mathrm{m}^{-2}$ & \\
\hline$H$ & Human community size & 1000 & Individual & \\
\hline$\mu_{H}$ & Per capita natural death rate of humans & $4 \times 10^{-5}$ & Day $^{-1}$ & \\
\hline$\mu_{P}$ & Per capita natural death rate of adult parasites & $5.5 \times 10^{-4}$ & Day $^{-1}$ & 19 and 25 \\
\hline$\mu_{S}$ & Per capita natural death rate of snails & $2.7 \times 10^{-3}$ & Day $^{-1}$ & 19 \\
\hline$d_{S}$ & Disease-induced death rate of snails & $1.7 \times 10^{-2}$ & Day $^{-1}$ & 19 \\
\hline$\delta$ & Exit-from-prepatency rate in snails & $6.7 \times 10^{-2}$ & Day $^{-1}$ & 19 and 25 \\
\hline$r$ & Cercariae releasing rate by one snail & 700 & Cercaria $\times$ snail $^{-1}$ & 3 and 19 \\
\hline$\alpha$ & Disease-induced death rate of humans per unit of parasite burden & $0,1.1 \times 10^{-7}$ & Day $^{-1}$ & 26 \\
\hline$k$ & Clumping parameter of parasite distribution & 0.243 & - & 19 \\
\hline$\gamma$ & Rate of competition for resources among snails & $10^{-2}$ & $\mathrm{~m}^{2} \times$ snail $^{-1}$ & 27 \\
\hline$\nu$ & Intrinsic natality rate of snails & 0.7 & $\mathrm{Day}^{-1}$ & 21 \\
\hline$\chi$ & Per capita rate of infection of snails & Variable & Parasite $^{-1} \times$ day $^{-1}$ & \\
\hline$\beta$ & Per capita rate of infection of humans & Variable & Parasite $\times \mathrm{m}^{2} \times$ cercaria $^{-1} \times$ individual $^{-1} \times$ day $^{-1}$ & \\
\hline
\end{tabular}

TABLE I. Variables and parameters of the model: description, value, unit and references. 
The rates of human and snail infection $(\beta$ and $\chi)$ are the most difficult to be quantified. Note that both parameters are the product of several factors. The first includes the probability of cercarial survival inside the human host and of maturation into the adult form of the parasite; the latter includes the number of produced eggs and their probability of reaching the aquatic environment, developing into miracidia and successfully infecting a susceptible snail. Due to the complexity associated with the definition of these parameters, we conduct a bifurcation analysis over a large parametric range.

\section{RESULTS}

\section{A. Equilibria and stability}

The nonlinear analysis of the system is performed under the assumption that the extra human mortality rate induced by one adult parasite is much smaller than the other parameters, so that it can be set to zero. Thus we study the following simplified system:

$$
\left\{\begin{array}{l}
\dot{N}=\mu_{H}(H-N) \\
\dot{P}=\beta r I N-\left(\mu_{H}+\mu_{P}\right) P \\
\dot{S}=\nu S[1-\gamma(S+E+I)]-\mu_{S} S-\chi P S \\
\dot{E}=\chi P S-\left(\mu_{S}+d_{S}\right) E-\delta E \\
\dot{I}=\delta E-\left(\mu_{S}+d_{S}\right) I .
\end{array}\right.
$$

Because the first equation is not coupled to the rest of system (7), it is sufficient to analyze the sub-system with state vector $X=[P, S, E, I]$. Setting $\dot{X}=0$ and $N=H$ provides two equilibria:

- A parasite-free equilibrium $X_{0}=\left(P_{0}, S_{0}, E_{0}, I_{0}\right)$, given by:

$$
X_{0}=\left\{\begin{array}{l}
S_{0}=\frac{\nu-\mu_{S}}{\nu \gamma} \\
P_{0}=0, E_{0}=I_{0}=0 .
\end{array}\right.
$$

- An endemic equilibrium $X_{+}=\left(P_{+}, S_{+}, E_{+}, I_{+}\right)$, given by:

$$
X_{+}=\left\{\begin{array}{l}
P_{+}=\frac{\beta r H}{\mu_{H}+\mu_{P}} I_{+} \\
S_{+}=\frac{\left(\mu_{H}+\mu_{P}\right)\left(\mu_{S}+d_{S}+\delta\right)\left(\mu_{S}+d_{S}\right)}{\chi \beta r H \delta} \\
E_{+}=\frac{\mu_{S}+d_{S}}{\delta} I_{+} \\
I_{+}=\frac{\frac{\nu-\mu_{S}}{\nu \gamma}-S_{+}}{\frac{\mu_{S}+d_{S}}{\delta}+\frac{\chi \beta r H}{\nu \gamma\left(\mu_{H}+\mu_{P}\right)}+1}
\end{array}\right.
$$

The parasite-free equilibrium $X_{0}$ is feasible because $\nu>\mu_{S}$. The endemic equilibrium $X_{+}$is feasible $\left(I_{+}>0\right)$ iff $S_{0}>S_{+}$. As for the stability of the parasite-free equilibrium, it is easy to prove that $X_{0}$ is stable if $X_{+}$is unfeasible, unstable otherwise (see Appendix A). This change of stability corresponds to a transcritical bifurcation. ${ }^{29}$ Its parametric expression represents the condition for which the Jacobian matrix associated with $X_{0}$ has an eigenvalue equal to zero, and it is given by

$$
\frac{\nu-\mu_{S}}{\nu \gamma}=\frac{\left(\mu_{H}+\mu_{P}\right)\left(\mu_{S}+d_{S}+\delta\right)\left(\mu_{S}+d_{S}\right)}{\chi \beta r H \delta} .
$$

Via stability analysis, it is also possible to prove that for some parametric conditions the endemic equilibrium $X_{+}$is unstable and there exists a stable limit cycle. This occurs via a supercritical Hopf bifurcation, whose expression is worked out analytically in Appendix B.

\section{B. Model simulations and bifurcation analysis}

Some representative temporal patterns of mean worm burden dynamics $P(t) / H$ obtained via simulation are shown in Figs. 3(a)-3(c). The solutions vary from disease-free to stationary endemic to periodic, this last displaying cycles characterized by fast increase and slow decrease of the mean worm burden, with a period of about $3 \mathrm{yr}$. The simulated parasite abundances are in good agreement with actual average burden data, as estimated from a study on a group of students and farmers in a village of south-western Sichuan, in China. ${ }^{11}$ These realistic patterns are detectable in a large range of the parameter space for both stationary and periodic
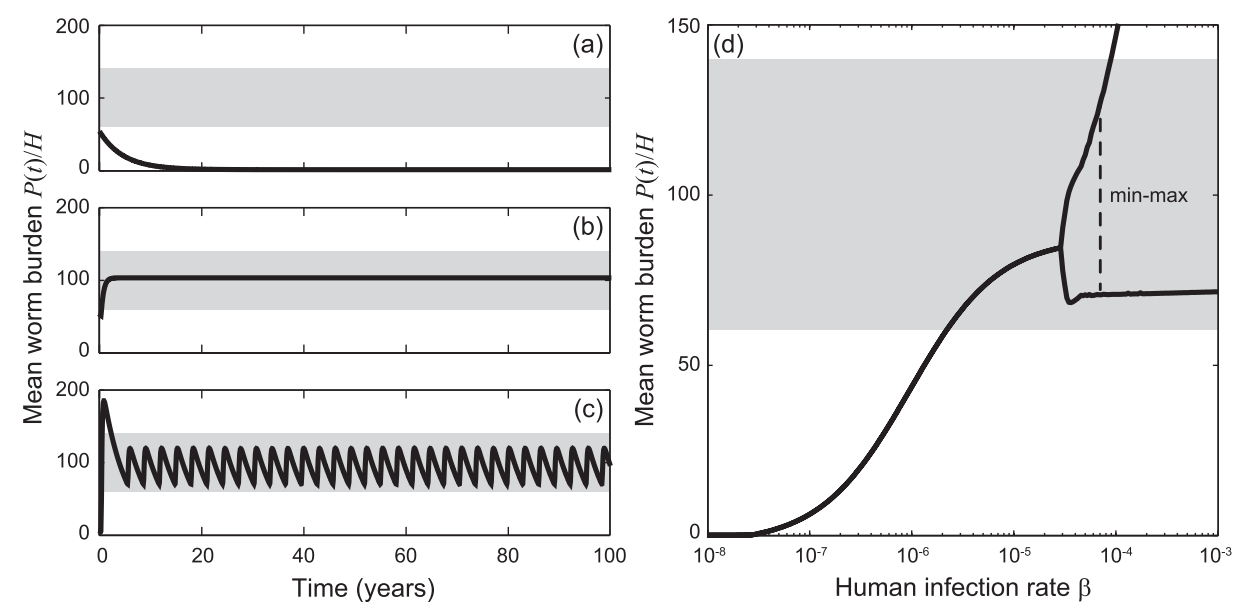

FIG. 3. Temporal patterns of mean worm burden and 1D bifurcation diagram. Panels (a)-(c) show different temporal patterns of mean worm burden $P(t) / H$. The infection rates are set as: (a) $\beta=3 \times 10^{-8}, \chi=3 \times 10^{-8}$, (b) $\beta=10^{-5}, \chi=6 \times 10^{-6}$, and (c) $\beta=6 \times 10^{-5}, \chi=8 \times 10^{-6}$. The $1 \mathrm{D}$ bifurcation diagram (d) is computed for $\chi=8 \times 10^{-6} /$ parasite/day. The grey shading represents the interval between lower and upper bounds of typical mean worm burden values (60-140 parasites per host) as estimated by Liang et al. ${ }^{11}$ Units and all other parameters as in Table I. 


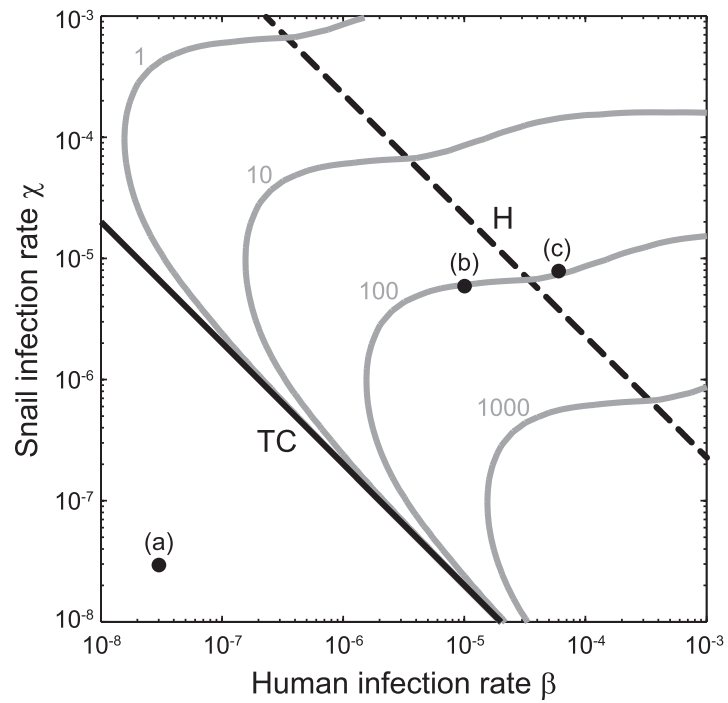

FIG. 4. Bifurcation curves of model (7) and level curves of time-averaged mean worm burden in the parameter space $(\beta, \chi)$. The black solid line represents the transcritical bifurcation curve (TC) that separates the region where the disease-free equilibrium $X_{0}$ is stable from the region where the endemic equilibrium $X_{+}$is feasible and stable. The black dashed line represents the Hopf bifurcation curve $(\mathrm{H})$ that delimits the region where the model displays stable periodic solutions. Grey lines represent the level curves of the mean worm burden for $\bar{P} / H=1,10,100,1000$ parasites/individual. Points (a)-(b)-(c) represent the combination of parameters $(\beta, \chi)$ used for the simulations shown in the left panels of Fig. 3. All other parameters as in Table I.

solutions (Figs. 3(b) and 3(c)). Fig. 3(d) shows that the mean worm burden increases with $\beta$ and that periodic solutions can be found for large values of $\beta$.

The bifurcation diagram of the model in the parameter space $(\beta, \chi)$ is shown in Fig. 4. For low values of the two infection rates (e.g., point (a) in Fig. 4), the disease-free equilibrium is stable and parasites are not able to establish in the population. For intermediate values of $\beta$ and $\chi$ (point (b)), the endemic equilibrium becomes feasible and stable via the transcritical bifurcation (TC) identified by Eq. (10). For larger values of the two infection rates (point (c)), the endemic equilibrium becomes unstable and periodic solutions originate via a supercritical Hopf bifurcation $(\mathrm{H})$, whose analytical derivation is reported in Appendix B. Fig. 4 also shows the level curves of the mean worm burden $\bar{P} / H$, with $\bar{P}$ being the time-averaged number of adult parasites evaluated after transient dynamics vanish. We can notice that mean worm burden increases with $\beta$ and, near the transcritical bifurcation, small variations of this parameter can lead to great variations of parasite abundance. In addition, for high values of $\beta$, the mean worm burden decreases with the snail infection rate $\chi$, while for low values of $\beta$ it is highest for intermediate values of $\chi$.

It is obviously possible to relax the hypothesis of no disease-induced human mortality $(\alpha=0)$ to see whether a small $\alpha>0$ changes the bifurcation structure of model (7). Figs. 5(a)-5(c) show some simulations obtained with a value of $\alpha>0$ similar to that recorded in Sudan. ${ }^{26}$ This induces variations in the population size of human hosts because of disease-induced mortality (Eq. (6)). In addition, Fig. 5(d) shows that, for small $\alpha$, the pattern of bifurcation is unchanged with both transcritical and Hopf bifurcations occurring, while for larger $\alpha$ periodic solutions are not possible. Also, the mean worm burden increases with $\alpha$ up to a certain value, then it decreases because of the severity of the disease. However, this happens for values of $\alpha$ far from realistic ones (at least one order of magnitude larger than the value estimated in $\operatorname{Sudan}^{26}$ ).

\section{Periodically forced model}

The ecology of a variety of snail habitats has been studied in several works, ${ }^{2,21}$ and many recent studies have been devoted to analyzing the relation between snail dynamics, environmental conditions, and schistosomiasis transmission. ${ }^{6,30}$ In particular, these studies have pointed out that the snail reproduction rate can vary a lot with the season. Therefore, in this section we investigate a more realistic, time-varying version of model (7) in which the reproduction of snails is assumed to vary periodically during the year, i.e.,

$$
\nu(t)=\nu_{0}\left[1+\epsilon \sin \frac{2 \pi t}{365}\right] .
$$

There exists an extensive literature on epidemiological models in seasonally varying environments. ${ }^{31-33}$ Here, however, the situation is more complex, because endogenously driven oscillations can be produced even in a constant environment for some values of $\nu_{0}$ (see Fig. 6(a), black solid line). Quite complex functioning modes can thus be expected
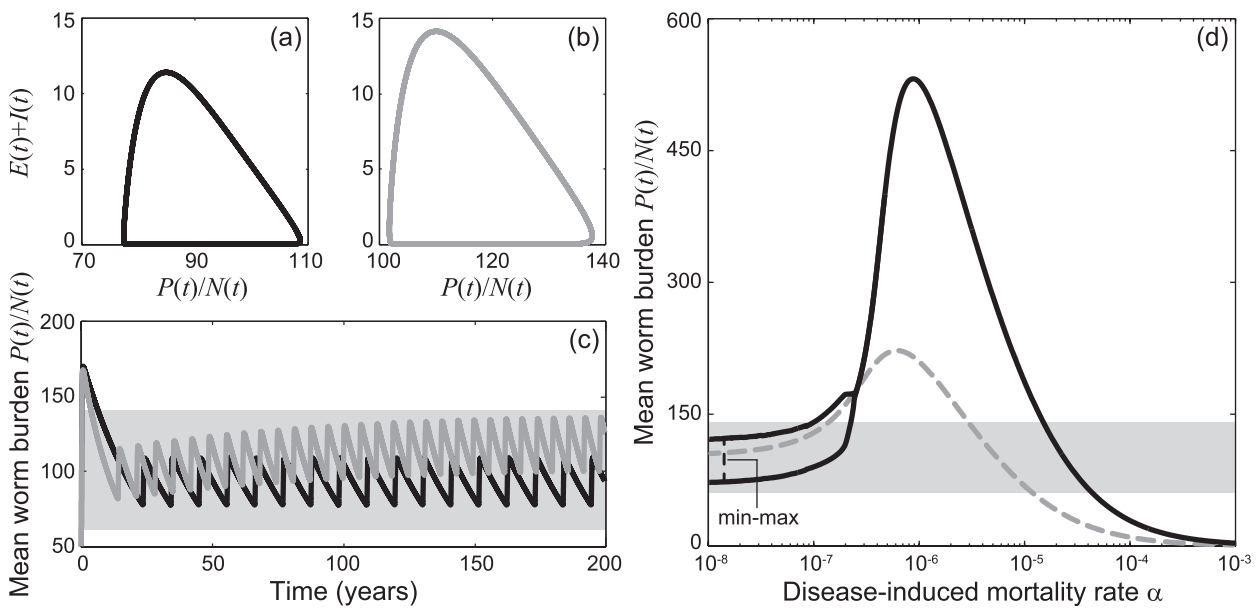

FIG. 5. Simulation of the model with nonvanishing disease-induced mortality and corresponding $1 \mathrm{D}$ bifurcation diagram. Numerical solutions of model (6) showing limit cycles obtained with (a) $\alpha=0$ (point (c) in Fig. 4), or (b) $\alpha=1.1 \times 10^{-7} /$ day and $k=0.243$. (c) Temporal patterns of mean worm burden $P(t) / N(t)$ for the two cases (black: $\alpha=0$; grey: $\alpha>0$ ). (d) Mean worm burden $P / N$ as a function of $\alpha$, for $\beta$ and $\chi$ as in points (b) (dashed line) and (c) (solid line) of Fig. 4. Grey shading as in Fig. 3. All other parameters as in Table I. 

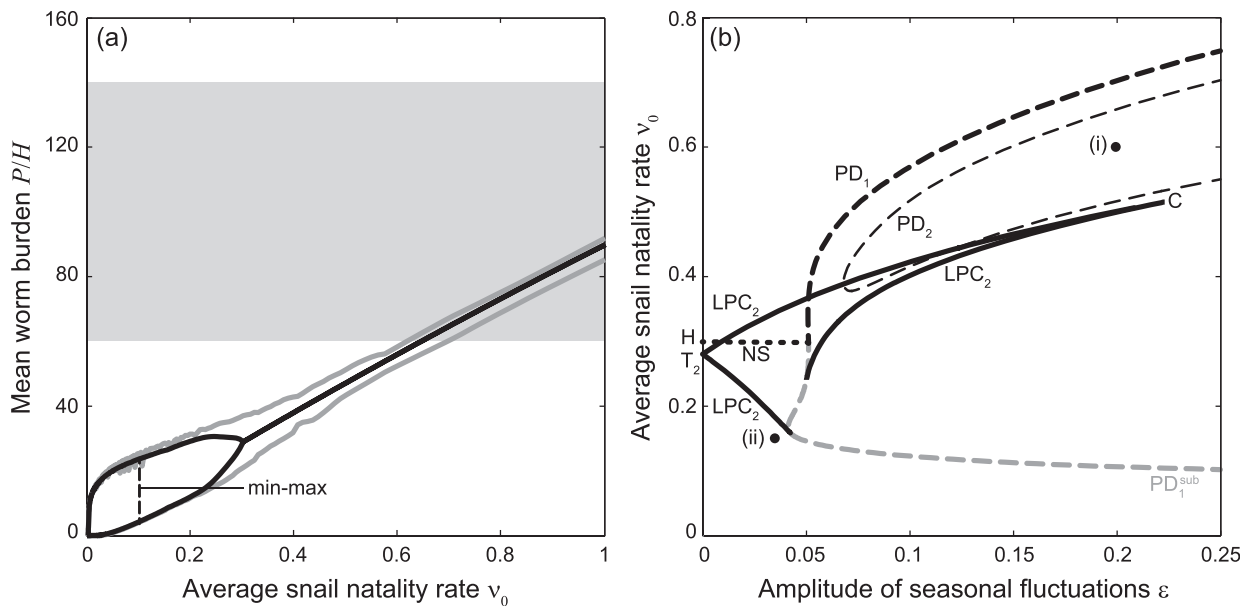

FIG. 6. Investigation of the timevarying version of model (7). (a) $1 \mathrm{D}$ bifurcation diagram computed for $\epsilon=0$ (black line) or $\epsilon=0.1$ (grey line). Grey shading as in Fig. 3. (b) Bifurcation diagram in the parameter space $\left(\epsilon, \nu_{0}\right)$ displaying supercritical $\left(\mathrm{PD}_{1}, \mathrm{PD}_{2}\right)$ and subcritical $\left(\mathrm{PD}_{1}^{\mathrm{sub}}\right)$ period-doubling bifurcations, Neimark-Sacker bifurcation (NS), and tangent of cycles bifurcation $\left(\mathrm{LPC}_{2}\right)$. Diagrams are computed for $\beta=10^{-5}$ and $\chi=10^{-5}$. All other parameters as in Table I. when $\nu$ is allowed to vary periodically over time, as it has been found for predator-prey ecological models in seasonally forced environments. ${ }^{34} \mathrm{We}$ explore the full complexity of the temporal patterns of disease severity and prevalence under seasonal snail ecology using software that implements continuation techniques, e.g., LOCBIF $^{35}$ or MATCONT. ${ }^{36} \mathrm{~A}$ simplified sketch of the bifurcation diagram in the parameter space $\left(\epsilon, \nu_{0}\right)$ is shown in Fig. 6(b).

Above the Hopf bifurcation point $\mathrm{H}$ on the $\nu_{0}$-axis, the unperturbed system reaches a stable equilibrium, while below that point the attractor is a limit cycle (with period of approximately $1.4 \mathrm{yr}$ close to the bifurcation). Therefore, a Neimark-Sacker bifurcation curve is rooted at point $\mathrm{H}$ : while crossing the curve from above, the limit cycle of the perturbed system becomes unstable and a stable torus arises. The two curves $\mathrm{LPC}_{2}$ merging in $\mathrm{T}_{2}$ are limit-point-of-cycle bifurcations that involve solutions with period two years. They are expected to emerge in the seasonally perturbed system from where, at $\epsilon=0$, the stable periodic solution has period two years. ${ }^{37}$ There are also several codimension-2 bifurcation points, only a few of which are shown in Fig. 6(b). The strong resonance 1:2 point at the junction of NS and $\mathrm{PD}_{1}$ or the generalized period doubling point that connects the lower $\mathrm{LPC}_{2}$ curve to $\mathrm{PD}_{1}$ are both expected and well-described in other bifurcation studies involving predator-prey models (such as Ref. 38). We notice that, despite the many similarities between our sketched diagram and the one obtained in those studies (see Fig. 1 in Ref. 38), here we also detect codimension- 2 points of different kind, such as the cusp $\mathrm{C}$ in Fig. 6(b). Nevertheless, we do not discuss them in detail here because they occur in parametric regions that are not very focal to our epidemiologically oriented analysis.

There exist two chaotic regions in the parameter space, which originate from different routes: the cascade of perioddoubling bifurcations (e.g., point (i) in Fig. 6(b)) and the torus breakdown (exemplified by point (ii) in Fig. 6(b)). These two kinds of strange attractors are shown in Fig. 7 together with their so-called peak-to-peak maps ${ }^{39}$ and the power spectra of the mean worm burden time series. As expected, the peak-to-peak map forms a relatively smooth curve in the case of the period-doubling route to chaos, and a more complex structure that is reminiscent of a torus in the case of torus breakdown. ${ }^{39}$ Furthermore, spectral analysis shows pronounced peaks that represent the characteristic periodicity of the cycle/torus from which each chaotic attractor originated. However, in both cases harmonic and subharmonic peaks emerge together with a continuous spectrum of frequencies, especially in the first route.

\section{DISCUSSION AND CONCLUSIONS}

In this paper, we have proposed a new model of schistosomiasis dynamics that, being of intermediate complexity, is both realistic and amenable to a thorough analysis of its temporal patterns. It neglects the dynamics of the larval stages of schistosomes, which have a much shorter lifespan than the adult parasites, but accounts for the population dynamics of snails and the parasite burden inside the human host. There exist several mathematical formulations to describe this infectious disease in the literature. However, our approach shows that it is possible to qualitatively reproduce both the intra- and inter-annual variability of prevalence patterns observed in many endemic regions via a simple, classical SEI-like model for snails.

We have first found the existence and stability conditions for the disease-free and endemic equilibria under the assumption that the extra human mortality rate induced by one adult parasite is negligible. Considering a time-constant environment for snails, we have demonstrated that the instability of the disease-free equilibrium corresponds to the existence of a unique endemic equilibrium (transcritical bifurcation), which can lose stability under some parametric conditions (supercritical Hopf bifurcation). The results imply that the basic mechanisms included in our model can destabilize the system, so that periodic interannual fluctuations can arise.

The inclusion of logistic recruitment for the snail population and of a prepatency period (incorporated via the Exposed compartment) leads to complex and realistic patterns. We have analyzed the whole range of model behaviors in the parameter space of the human and snail infection rates, which we choose as most significant and subject to uncertainty and wide variations (other parameters are set to values obtained from the relevant literature). Besides qualitative changes in system dynamics, different combinations of 

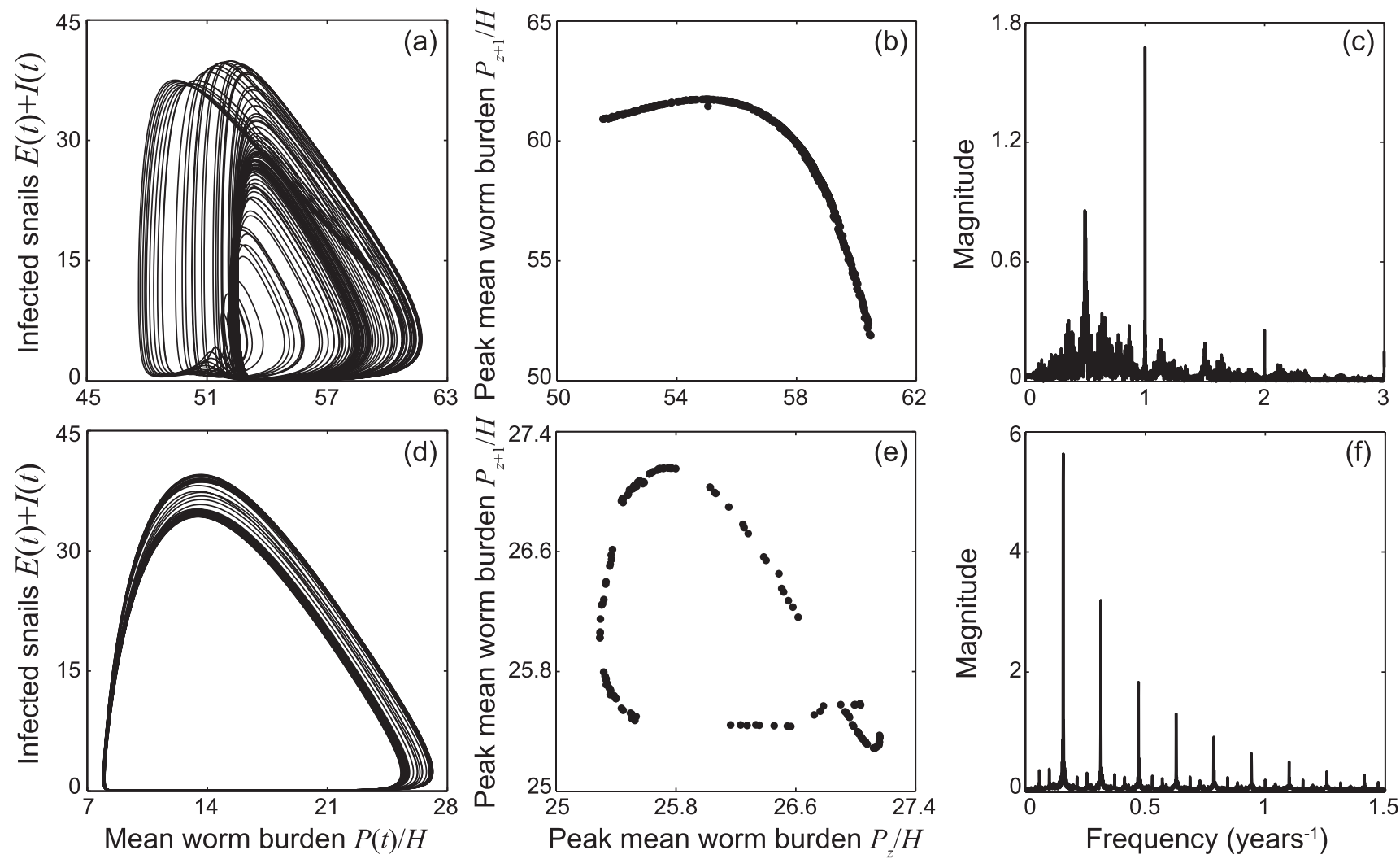

FIG. 7. Strange attractors of model (7). (a) State reconstruction of the mean worm burden and infected snails, (b) peak-to-peak map, and (c) power spectrum of the mean worm burden time series for the strange attractor originated via cascade of period-doubling bifurcations (point (i) of Fig. 6; $\epsilon=0.2, \nu_{0}=0.6 /$ day). (d)-(e)-(f) As in (a)-(b)-(c) for the strange attractor originated via torus breakdown (point (ii) of Fig. 6; $\epsilon=0.035, \nu_{0}=0.15 /$ day). Time-varying model's behaviors are investigated for $\beta=10^{-5}$ and $\chi=10^{-5}$. All other parameters as in Table I.

human/snail infection rates lead to remarkable quantitative differences in the mean parasite burden of human hosts. Including a non-vanishing parasite-induced mortality in the human host may change the quantitative behavior of the model, but for realistic values of this mortality the temporal patterns remain qualitatively unchanged.

We have also investigated the effect of seasonal fluctuations of snail demography, which is strongly dependent on environmental conditions. Results suggest that environmental variability has an important role in generating the disease dynamical patterns over time. Wide intra-annual fluctuations can lead to sub-harmonic oscillations and chaotic behaviors. Bifurcation analysis and numerical simulations thus show that the basic mechanisms included in our model are able to reproduce behaviors also found in real data, including periodic, quasi-periodic, and chaotic dynamics. This makes our model a useful tool to better investigate the impact of anthropic and environmental changes on the severity and prevalence of schistosomiasis. Although our approach is largely theoretical, the intermediate complexity of our model makes it also a promising building brick towards a realistic framework for the study not only of temporal but also of spatial patterns of schistosomiasis dynamics. This model could be the basis for the development of a new spatially explicit modeling framework, accounting, e.g., for both human mobility and geographical interconnections between snail populations through hydrological networks. ${ }^{40,41}$

\section{ACKNOWLEDGMENTS}

M.C., M.G., and R.C. acknowledge support from the Doctoral School of Politecnico di Milano. L.M. and A.R. acknowledge the Swiss Agency for Development and Cooperation for support through the project "Santé des populations: maladies d'origine hydrique."

\section{APPENDIX A: TRANSCRITICAL BIFURCATION CURVE}

From the linearization of system (7), we can write the Jacobian matrix $J$

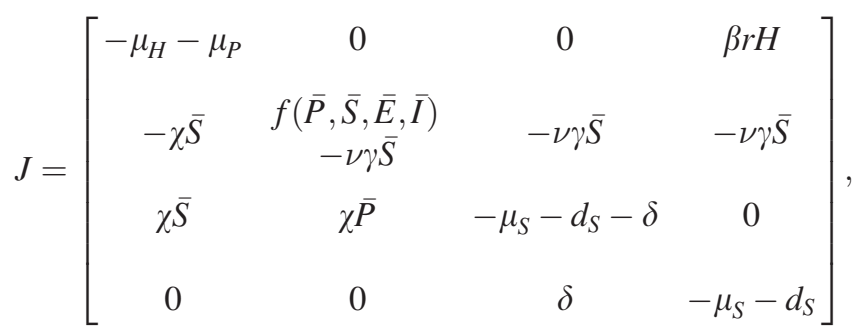

where a superscript bar indicates state variables at equilibrium and $f(\bar{P}, \bar{S}, \bar{E}, \bar{I})$ is given by

$$
f(\bar{P}, \bar{S}, \bar{E}, \bar{I})=\nu[1-\gamma(\bar{S}+\bar{E}+\bar{I})]-\mu_{S}-\chi \bar{P} .
$$

Evaluating $J$ at the disease free equilibrium $X_{0}$, we derive that one eigenvalue is $\lambda_{1}=\mu_{S}-\nu$, that is always negative; the other three solve the equation 


$$
\begin{aligned}
\operatorname{det}\left[\begin{array}{ccc}
\lambda+\mu_{H}+\mu_{P} & 0 & -\beta r H \\
-\chi S_{0} & \lambda+\mu_{S}+d_{S}+\delta & 0 \\
0 & -\delta & \lambda+\mu_{S}+d_{S}
\end{array}\right] \\
=\left(\lambda+\mu_{H}+\mu_{P}\right)\left(\lambda+\mu_{S}+d_{S}+\delta\right)\left(\lambda+\mu_{S}+d_{S}\right) \\
-\chi \beta r H \delta \frac{\nu-\mu_{S}}{\nu \gamma}=0,
\end{aligned}
$$

which reads

$$
f(\lambda)=a_{0} \lambda^{3}+a_{1} \lambda^{2}+a_{2} \lambda+a_{3}=0,
$$

with

$$
\begin{gathered}
a_{0}=1, \\
a_{1}=2 \mu_{S}+2 d_{S}+\delta+\mu_{H}+\mu_{P}, \\
a_{2}=\left(\mu_{H}+\mu_{P}\right)\left(2 \mu_{S}+2 d_{S}+\delta\right)+\left(\mu_{S}+d_{S}+\delta\right)\left(\mu_{S}+d_{S}\right), \\
a_{3}=\left(\mu_{H}+\mu_{P}\right)\left(\mu_{S}+d_{S}+\delta\right)\left(\mu_{S}+d_{S}\right)-\chi \beta r H \delta \frac{\nu-\mu_{S}}{\nu \gamma} .
\end{gathered}
$$

Since $a_{0}, a_{1}$, and $a_{2}$ are always positive, the changes of sign between consecutive nonzero coefficients $a_{i}$ are at most 1 if $a_{3}<0$. In that case, there is one positive eigenvalue. Note that the coefficients of

$$
f(-\lambda)=-a_{0} \lambda^{3}+a_{1} \lambda^{2}-a_{2} \lambda+a_{3}=0
$$

do change sign either twice or three times if $a_{3}<0$ or $a_{3}>0$, respectively. By Descartes' rule of signs, there are at most two or three negative eigenvalues for $J\left(X_{0}\right)$. Oscillations around $X_{0}$ are not possible because of the positivity of the system, thus no complex roots are expected for $f(\lambda)$. We can conclude that the condition $a_{3}<0$ is the stability condition of the disease-free equilibrium. Since $a_{3}<0$ is also the condition for the positivity of the endemic equilibrium $X_{+}$(see Eq. (9)), we obtain Eq. (10) for the transcritical bifurcation curve.

\section{APPENDIX B: HOPF BIFURCATION CURVE}

We evaluate the Jacobian matrix (A1) at $X_{+}$and write the characteristic matrix

$$
\begin{aligned}
& \lambda I-J\left(X_{+}\right) \\
& =\left[\begin{array}{cccc}
\lambda+\mu_{H}+\mu_{P} & 0 & 0 & -\beta r H \\
\chi S_{+} & \lambda+\nu \gamma S_{+} & \nu \gamma S_{+} & \nu \gamma S_{+} \\
-\chi S_{+} & -\chi P_{+} & \lambda+\mu_{S}+d_{S}+\delta & 0 \\
0 & 0 & -\delta & \lambda+\mu_{S}+d_{S}
\end{array}\right] .
\end{aligned}
$$

Therefore, the characteristic equation $\operatorname{det}\left(\lambda I-J\left(X_{+}\right)\right)$ $=0$ is a fourth order polynomial equation of the form

$$
f(\lambda)=\lambda^{4}+b_{1} \lambda^{3}+b_{2} \lambda^{2}+b_{3} \lambda+b_{4}=0
$$

where

$$
\begin{gathered}
b_{1}=\mu_{H}+\mu_{P}+2 \mu_{S}+2 d_{S}+\delta+\nu \gamma S_{+}, \\
b_{2}=\nu \gamma S_{+}\left(2 \mu_{S}+2 d_{S}+\delta+\chi P_{+}\right)+\left(\mu_{S}+d_{S}+\delta\right) \\
\times\left(\mu_{S}+d_{S}\right)+\left(\mu_{H}+\mu_{P}\right)\left(\nu \gamma S_{+}+2 \mu_{S}+2 d_{S}+\delta\right), \\
b_{3}=\nu \gamma S_{+}\left[\left(\mu_{S}+d_{S}+\delta\right)\left(\mu_{S}+d_{S}\right)+\chi P_{+}\left(\delta+\mu_{S}+d_{S}\right.\right. \\
\left.\left.+\mu_{H}+\mu_{P}\right)\right]+\left(\mu_{H}+\mu_{P}\right)\left[\nu \gamma S_{+}\left(2 \mu_{S}+2 d_{S}+\delta\right)\right. \\
\left.+\left(\mu_{S}+d_{S}+\delta\right)\left(\mu_{S}+d_{S}\right)\right]-\beta r H \chi \delta S_{+}, \\
b_{4}=\left(\mu_{H}+\mu_{P}\right)\left\{\nu \gamma S _ { + } \left[\left(\mu_{S}+d_{S}+\delta\right)\left(\mu_{S}+d_{S}\right)+\chi \delta P_{+}\right.\right. \\
\left.\left.+\left(\mu_{S}+d_{S}\right) \chi P_{+}\right]\right\}+\beta r H \delta \chi S_{+}\left(\chi P_{+}-\nu \gamma S_{+}\right) .
\end{gathered}
$$

To analyze the stability of the endemic equilibrium $X_{+}$ we construct the Routh-Hurwitz table

\begin{tabular}{ccc}
\hline \hline 1 & $b_{2}$ & $b_{4}$ \\
$b_{1}$ & $b_{3}$ & 0 \\
$-\frac{1}{b_{1}}\left(b_{3}-b_{1} b_{2}\right)$ & $-\frac{1}{b_{1}}\left(-b_{1} b_{4}\right)$ & 0 \\
$-\frac{b_{1}}{b_{1} b_{2}-b_{3}}\left(b_{1} b_{4}-b_{3} \frac{b_{1} b_{2}-b_{3}}{b_{1}}\right)$ & 0 & 0 \\
\hline
\end{tabular}

According to the Routh-Hurwitz criterion, the equilibrium is stable iff

$$
\left\{\begin{array}{l}
b_{1}>0, \\
b_{1} b_{2}>b_{3}, \\
b_{1} b_{2} b_{3}<b_{1}^{2} b_{4}+b_{3}^{2} .
\end{array}\right.
$$

As the first condition is verified as long as $X_{+}$is feasible, the conditions for a supercritical Hopf bifurcation of $X_{+}$to occur are

$$
\left\{\begin{array}{l}
b_{1} b_{2}>b_{3}, \\
b_{1} b_{2} b_{3}=b_{1}^{2} b_{4}+b_{3}^{2} .
\end{array}\right.
$$

In fact, for these parameter values the Jacobian matrix $J\left(X_{+}\right)$would have a pair of purely imaginary eigenvalues (see Ref. 42).

${ }^{1}$ L. Chitsulo, D. Engels, A. Montresor, and L. Savioli, "The global status of schistosomiasis and its control," Acta Trop. 77, 41-51 (2000).

${ }^{2}$ WHO Expert Committee, "Prevention and control of schistosomiasis and soil-transmitted helminthiasis," Technical Report (WHO, 2002).

${ }^{3}$ D. G. Colley, A. L. Bustinduy, W. E. Secor, and C. H. King, "Human schistosomiasis," Lancet 383, 2253-2264 (2014).

${ }^{4}$ K. Tzanetou, G. Adamis, E. Andipa, C. Zorzos, K. Ntoumas, K. Armenis, G. Kontogeorgos, E. Malamou-Lada, and P. Gargalianos, "Urinary tract Schistosoma haematobium infection: A case report," J. Travel Med. 14, 334-337 (2007).

${ }^{5}$ A. Samie, D. J. Nchachi, C. L. Obi, and E. O. Igumbor, "Prevalence and temporal distribution of Schistosoma haematobium infections in the Vhembe district, Limpopo Province, South Africa," African J. Biotechnol. 9, 7157-7164 (2010), see http://www.academicjournals.org/journal/AJB/ article-abstract/D5E7AA124489.

${ }^{6}$ Z. Xue, M. Gebremichael, R. Ahmad, M. L. Weldu, and A. C. Bagtzoglou, "Impact of temperature and precipitation on propagation of intestinal schistosomiasis in an irrigated region in Ethiopia: suitability of satellite datasets," Trop. Med. Int. Health 16, 1104-1111 (2011). 
${ }^{7}$ R. M. Anderson and R. M. May, Infectious Diseases of Humans: Dynamics and Control (Oxford University Press, Oxford, 1992).

${ }^{8}$ K. M. Mitchell, F. Mutapi, T. Mduluza, N. Midzi, N. J. Savill, and M. E. J. Woolhouse, "Predicted impact of mass drug administration on the development of protective immunity against Schistosoma haematobium," PLoS Negl. Trop. Dis. 8, e3059 (2014).

${ }^{9}$ S. Tour, Y. Zhang, E. Bosqu-Oliva, C. Ky, A. Ouedraogo, A. Koukounari, A. F. Gabrielli, B. Sellin, J. P. Webster, and A. Fenwick, "Two-year impact of single praziquantel treatment on infection in the national control programme on schistosomiasis in Burkina Faso," Bull. World Health Organ. 86, 780-787 (2008).

${ }^{10}$ M. S. Chan, H. L. Guyatt, D. A. P. Bundy, M. Booth, A. J. C. Fulford, and G. F. Medley, "The development of an age structured model for schistosomiasis transmission dynamics and control and its validation for Schistosoma mansoni," Epidemiology Infect. 115, 325-344 (1995).

${ }^{11}$ S. Liang, R. C. Spear, E. Seto, A. Hubbard, and D. Qiu, "A multi-group model of Schistosoma japonicum transmission dynamics and control: model calibration and control prediction," Trop. Med. Int. Health 10, 263-278 (2005).

${ }^{12}$ J. Xiang, H. Chen, and H. Ishikawa, "A mathematical model for the transmission of Schistosoma japonicum in consideration of seasonal water level fluctuations of Poyang Lake in Jiangxi, China," Parasitol. Int. 62, 118-126 (2013).

${ }^{13}$ D. Gurarie and C. H. King, "Heterogeneous model of schistosomiasis transmission and long-term control: The combined influence of spatial variation and age-dependent factors on optimal allocation of drug therapy," Parasitology 130, 49-65 (2005).

${ }^{14} \mathrm{P}$. Zhang, Z. Feng, and F. Milner, "A schistosomiasis model with an agestructure in human hosts and its application to treatment strategies," Math. Biosci. 205, 83-107 (2007).

${ }^{15} \mathrm{~N}$. G. Hairston, "On the mathematical analysis of schistosome populations," Bull. World Health Organ. 33, 45-62 (1965), see http:// www.ncbi.nlm.nih.gov/pmc/articles/PMC2475813/.

${ }^{16} \mathrm{G}$. Macdonald, "The dynamics of helminth infections, with special reference to schistosomes," Trans. R. Soc. Trop. Med. Hyg. 59, 489-506 (1965).

${ }^{17}$ R. M. Anderson and R. M. May, "Regulation and stability of host-parasite population interactions. I. Regulatory processes,” J. Anim. Ecol. 47, 219-247 (1978).

${ }^{18}$ Z. Feng, C.-C. Li, and F. A. Milner, "Schistosomiasis models with density dependence and age of infection in snail dynamics," Math. Biosci. 177-178, 271-286 (2002).

${ }^{19}$ Z. Feng, A. Eppert, F. A. Milner, and D. J. Minchella, "Estimation of parameters governing the transmission dynamics of schistosomes," Appl. Math. Lett. 17, 1105-1112 (2004).

${ }^{20}$ C. Castillo-Chavez, Z. Feng, and D. Xu, "A schistosomiasis model with mating structure and time delay," Math. Biosci. 211, 333-341 (2008).

${ }^{21}$ T. D. Mangal, S. Paterson, and A. Fenton, "Effects of snail density on growth, reproduction and survival of Biomphalaria alexandrina exposed to Schistosoma mansoni," J. Parasitol. Res. 2010, 2313-2319.

${ }^{22}$ J. Wu, R. Dhingra, M. Gambhir, and J. V. Remais, "Sensitivity analysis of infectious disease models: methods, advances and their application,” J. R. Soc. Interface 12, 1018 (2013).

${ }^{23}$ A. Guiro, S. Ouaro, and A. Traoré, "Stability analysis of a schistosomiasis model with delays," Adv. Differ. Equ. 2013, 1-15 (2013).
${ }^{24}$ L. Qi, J. an Cui, T. Huang, F. Ye, and L. Jiang, "Mathematical model of schistosomiasis under flood in Anhui Province," Abstr. Appl. Anal. 2014, 972189 (2014).

${ }^{25}$ B. Gryseels, K. Polman, J. Clerinx, and L. Kestens, "Human schistosomiasis," Lancet 368, 1106-1118 (2006).

${ }^{26}$ M. M. Kheir, I. A. Eltoum, A. M. Saad, M. M. Ali, O. Z. Baraka, and M. M. Ali Homeida, "Mortality due to schistosomiasis mansoni: a field study in Sudan," Am. J. Trop. Med. Hyg. 60, 307-310 (1999), see http:// www.ajtmh.org/content/60/2/307.long.

${ }^{27}$ E. Seto, S. Liang, D. Qiu, X. Gu, and R. C. Spear, "A protocol for geographically randomized snail surveys in schistosomiasis fieldwork using the global positioning system," Am. J. Trop. Med. Hyg. 64, 98-99 (2001), see http://www.ajtmh.org/content/64/1/98.long.

${ }^{28} \mathrm{G}$. Webbe, "The transmission of Schistosoma haematobium in an area of Lake Province, Tanganyika,” Bull. World Health Organ. 27, 59-85 (1962), http://www.ncbi.nlm.nih.gov/pmc/articles/PMC2555818/.

${ }^{29}$ Y. Kuznetsov, Elements of Applied Bifurcation Theory (Springer, 1995).

${ }^{30} \mathrm{~N}$. McCreesh and M. Booth, "The effect of increasing water temperatures on Schistosoma mansoni transmission and Biomphalaria pfeifferi population dynamics: An agent-based modelling study," PLoS ONE 9, e101462 (2014).

${ }^{31}$ Y. Kuznetsov and C. Piccardi, "Bifurcation analysis of periodic SEIR and SIR epidemic models," J. Math. Biol. 32, 109-121 (1994).

${ }^{32}$ R. Casagrandi, L. Bolzoni, S. A. Levin, and V. Andreasen, "The SIRC model and influenza A,” Math. Biosci. 200, 152-169 (2006).

${ }^{33}$ L. Righetto, R. Casagrandi, E. Bertuzzo, L. Mari, M. Gatto, I. RodriguezIturbe, and A. Rinaldo, "The role of aquatic reservoir fluctuations in longterm cholera patterns," Epidemics 4, 33-42 (2012).

${ }^{34}$ S. Rinaldi, S. Muratori, and Y. Kuznetsov, "Multiple attractors, catastrophes and chaos in seasonally perturbed predator-prey communities," Bull. Math. Biol. 55, 15-35 (1993).

${ }^{35}$ A. Khibnik, Y. Kuznetsov, V. Levitin, and E. Nikolaev, "Continuation techniques and interactive software for bifurcation analysis of ODEs and iterated maps," Physica D 62, 360-371 (1993).

${ }^{36}$ A. Dhooge, W. Govaerts, and Y. Kuznetsov, "MATCONT: A MATLAB package for numerical bifurcation analysis of ODEs," ACM Trans. Math. Softw. 29, 141-164 (2003).

${ }^{37}$ J. Guckenheimer and P. Holmes, Nonlinear Oscillations, Dynamical Systems, and Bifurcations of Vector Fields (Springer-Verlag, New York, NY, 1983).

${ }^{38}$ V. Witte, F. Rossa, W. Govaerts, and Y. Kuznetsov, "Numerical periodic normalization for codim 2 bifurcations of limit cycles: computational formulas, numerical implementation, and examples," SIAM J. Appl. Dyn. Syst. 12, 722-788 (2013).

${ }^{39}$ M. Candaten and S. Rinaldi, "Peak-to-peak dynamics: A critical survey," Int. J. Bifurcation Chaos 10, 1805-1819 (2000).

${ }^{40}$ D. Gurarie and E. Y. W. Seto, "Connectivity sustains disease transmission in environments with low potential for endemicity: modelling schistosomiasis with hydrologic and social connectivities," J. R. Soc. Interface 6, 495-508 (2009).

${ }^{41}$ J. Remais, "Modelling environmentally-mediated infectious diseases of humans: transmission dynamics of schistosomiasis in China," in Modelling Parasite Transmission and Control (Springer, 2010), pp. 79-98.

${ }^{42} \mathrm{~T}$. Asada and H. Yoshida, "Coefficient criterion for four-dimensional Hopf bifurcations: a complete mathematical characterization and applications to economic dynamics," Chaos, Solitons Fractals 18, 525-536 (2003). 